\title{
Patient-Reported Geriatric Symptoms as Risk Factors for Hospitalization and Emergency Department Visits
}

\author{
Anupam Chandra', Sarah J Crane ${ }^{1}$, Ericka E Tung', Gregory J Hanson ${ }^{1}$, Frederick \\ North $^{1}$, Stephen S Cha ${ }^{2}$, Paul Y Takahashi ${ }^{1}$ \\ ${ }^{1}$ Division of Primary Care Internal Medicine and ${ }^{2}$ Department of Health Sciences Research; Mayo Clinic, 200 \\ First Street SW, Rochester, MN 55905, USA
}

[Received December 06, 2013; Revised July 1, 2014; Accepted July 6, 2014]

\begin{abstract}
There is an urgent need to identify predictors of adverse outcomes and increased health care utilization in the elderly. The Mayo Ambulatory Geriatric Evaluation (MAGE) is a symptom questionnaire that was completed by patients aged 65 years and older during office visits to Primary Care Internal Medicine at Mayo Clinic in Rochester, MN. It was introduced to improve screening for geriatric conditions. We conducted this study to explore the relationship between self-reported geriatric symptoms and hospitalization and emergency department (ED) visits within 1 year of completing the survey. This was a retrospective cohort study of patients who completed the MAGE from April 2008 to December 2010. The primary outcome was an ED visit or hospitalization within 1 year. Predictors included responses to individual questions in the MAGE. Data were obtained from the electronic medical record and administrative records. Logistic regression analyses were performed from significant univariate factors to determine predictors in a multivariable setting. A weighted scoring system was created based upon the odds ratios derived from a bootstrap process. The sensitivity, specificity, and AUC were calculated using this scoring system. The MAGE survey was completed by 7738 patients. The average age was $76.2 \pm 7.68$ years and $57 \%$ were women. Advanced age, a self-report of worse health, history of 2 or more falls, weight loss, and depressed mood were significantly associated with hospitalization or ED visits within 1 year. A score equal to or greater than 2 had a sensitivity of 0.74 and specificity of 0.45 . The calculated AUC was 0.60 . The MAGE questionnaire, which was completed by patients at an outpatient visit to screen for common geriatric issues, could also be used to assess risk for ED visits and hospitalization within 1 year.
\end{abstract}

Key words: emergency department, geriatric screening, hospitalization

Aging is associated with a higher burden of disease, disability, and complex medical needs. In order to effectively address these concerns, screening strategies that identify issues that affect the quality of life and health outcomes in the elderly are needed. Health care providers are keenly interested in identifying predictors of adverse health outcomes such as emergency department (ED) visits and hospitalizations in the elderly. This interest, largely due to an increase in the aging population, is further driven by the Affordable Care Act which provides impetus to contain costs and reduce avoidable hospital readmissions [1]. Previous studies have examined predictors for increased health care utilization and adverse outcomes and introduced instruments, which incorporate factors such as medical complexity, self-rated health, previous utilization, and socioeconomic and functional status, to assess this risk in different settings [2-7]. Falls, urinary incontinence, vision and hearing difficulties, weight loss, depression, poor memory, and poor self-rate of health are common geriatric issues which deeply affect the quality of life in the elderly. Relatively little information is available on these geriatric syndromes as risk factors associated with hospitalization or ED visits, especially among the elderly living in the community.

*Correspondence should be addressed to: Anupam Chandra, MD, Mayo Clinic, 200 First Street SW, Rochester MN 55905, USA. Email: chandra.anupam@mayo.edu 
These conditions may place them at increased risk for adverse outcomes because they cause direct health decline or serve as markers for overall frailty [8].

The Mayo Ambulatory Geriatric Evaluation (MAGE) is a clinical instrument that was introduced in 2007 in the Division of Primary Care Internal Medicine (PCIM) at Mayo Clinic in Rochester, MN, to improve screening for common geriatric conditions in the outpatient environment. It was a questionnaire completed by patients aged 65 years and older during the office visit with their provider. It included questions related to vision, hearing, weight loss, mood, falls, incontinence, self-report of health, and memory. It was intended that a positive screen would be addressed during the visit by the patient's primary care provider. The purpose of this study was to examine the relationship between responses to the MAGE questionnaire and hospitalization and/or ED visits within 1 year.

\section{MATERIALS AND METHODS}

\section{Study design and subjects}

This was a retrospective cohort study of the records of patients aged 65 years and older. The study was approved by the Mayo Clinic Institutional Review Board. The setting was an academic internal medicine primary care practice at a single location in Rochester, MN. The records of patients who had completed the MAGE questionnaire survey as part of their clinical care process between April 2008 and December 2010 were reviewed. Those who had declined consent for research were excluded.

\section{MAGE instrument}

The MAGE questionnaire was developed by geriatricians in the Division of PCIM at Mayo Clinic in Rochester, $\mathrm{MN}$, to improve the current clinical practice in providing care to the geriatric population. The domains were selected to identify geriatric symptoms that were common and could potentially have significant effects on quality of life and outcomes and may be missed during a routine appointment. Questions for each domain were selected by consensus, drawing from clinical experience and expertise. The emphasis was to keep the questionnaire high yield, yet brief and simple, with minimal time burden to patients or providers. Completion of the survey was incorporated as the standardized rooming process for geriatric patients aged 65 and older using interpreters if needed. This was done either in the waiting room or the exam room prior to the provider visit. In this clinic, patients are required to check in 20 minutes prior to the visit with the provider and the survey was completed during that time. The rooming staff and providers were available to assist in completing the survey if patients and their families were unable to complete the survey on their own. No patients were excluded because of language barriers, age, cognitive status, or morbidity. This study is a retrospective analysis of this clinical process.

\section{Variables}

The primary outcome variables of hospitalization and/or ED visit within 1 year of completing the survey were obtained from the electronic medical record (EMR) and administrative data. Emergency department visits were determined by identifying the record of service provided in the ED. Patients deemed to have been hospitalized had a hospital admit note and dismissal summary entered in the EMR. Death information was obtained from the patient clinical record.

Responses to the individual questions on the MAGE, age, and gender were used as predictor variables. This information was obtained from the EMR. Age was reported both as a continuous and categorized variable. The survey included questions pertaining to unintentional weight loss in the last 3 months, history of 2 or more falls in the past year, being hurt in a fall in the past year, vision difficulty, hearing difficulty, being sad or depressed, urinary incontinence, incontinence severe enough for treatment, and self-rated health compared to peers. The clock draw test completed by the patient was not included for analysis due to lack of standardization in interpretation and charting.

\section{Analysis}

The most recently completed survey was used for individuals who completed multiple MAGE surveys over 3 years. Mean and standard deviation for continuous data or frequency (percentage) for categorical data were tabulated for each question on the MAGE survey. We used a logistic regression analysis on hospital admissions and ED visits to develop a scoring mechanism using the bootstrap method. This method drew repeated samples of the same size from 7738 patients with replacement 2000 times. The odds ratio (OR) of the bootstrap method was the average OR from these 2000 simulations. The score for each predictor came after rounding off the OR to an integer and included those that retained a 0.05 significance level over $50 \%$ of the time. Those with missing responses were excluded from analysis. 


\section{RESULTS}

During the specified time frame, 12,157 MAGE surveys were completed by 7738 patients. The average age was $76.2 \pm 7.68$ years and $57 \%$ were women. The descriptive results are summarized in Table 1.

Of 7738 patients, 2674 (34.6\%) had the combined outcome of hospitalizations or ED visits within 1 year of completing the MAGE survey. On unadjusted univariate analysis, a positive response to any of the questions in the MAGE survey was significantly associated with combined outcome (Table 2). Worse self-rated health and a history of 2 or more falls in the past year were the most significant univariate predictors. The average age of the group with the combined outcome of hospitalization or ED visit within 1 year was 78.05 years ( $S D \pm 7.87$ years) compared to 75.15 years ( $\mathrm{SD} \pm 7.39$ years) in the group without this outcome. In the group with the combined outcome, $9 \%$ rated their health as worse compared to $4 \%$ in the group without this outcome (OR 2.55; 95\% CI, 2.10-3.10). The other significant factor on univariate analysis was a history of 2 or more falls in the past year with an OR of 2.09 (95\% CI, 1.83-2.40).

Table 1. Demographics and results of the MAGE survey

\begin{tabular}{ll}
\hline Variable & Overall (N=7738) \\
\hline Age, y mean ( \pm SD) & $76.2( \pm 7.68)$ \\
Female, n (\%) & $4395(57)$ \\
Male, n (\%) & $3343(43)$ \\
MAGE Questions, n (\%) & $598(8)$ \\
History of weight loss in last 3 months & $962(12)$ \\
History of 2 or more falls in past year & $930(12)$ \\
History of being hurt in fall in past year & $1526(20)$ \\
Difficulty with vision & $3105(41)$ \\
Difficulty with hearing & $864(11)$ \\
History of being sad or depressed & $3147(41)$ \\
History of unintended urinary leakage & $626(9)$ \\
History of urinary leakage severe enough to seek treatment & \\
Rate your health compared to others in your age group & $3484(45)$ \\
$\quad$ Better & $3487(45)$ \\
Same & $432(6)$ \\
Worse & $335(4)$ \\
Missing &
\end{tabular}

MAGE: Mayo Ambulatory Geriatric Evaluation

In the multivariable model to determine predictors for combined outcome, age, gender, and responses to MAGE questions were included (Table 3). Age 80 or above was the most significant predictor with an OR of 2.29 (95\% CI, 1.98-2.64). Worse self-rated health remained a strong predictor associated with the combined outcome of ED visit or hospitalization within 1 year with an OR of 2.11 (95\% CI, 1.70-2.63). A history of 2 or more falls was also significant with an OR of 1.63 (95\% CI, 1.38-1.93). A history of weight loss or depressed mood was significant to a lesser extent. Gender, hearing loss, vision difficulties, incontinence, and history of being hurt in a fall did not remain significant on multivariable testing. Based upon the OR derived from the bootstrap method, a weighted scoring system was created with a single point each for age 70-74 years, history of weight loss, and depressed mood; and two points each for age 7579 years, age 80 years or above, worse self-rate of health, and a history of 2 or more falls. Participants could have a score from zero (none of the above) to a maximum of eight points. The sensitivity, specificity, and area under the curve were created using this scoring system to predict a combined outcome of hospitalizations or ED visits. The calculated area under the curve was 0.60 . A score $\geqslant 2$ had a sensitivity of 0.74 and specificity of 0.45 . The individual 
Table 2. Univariate predictors of the combined outcome of ED visit or hospital admission within 1 year

\begin{tabular}{|c|c|c|c|c|c|c|}
\hline \multirow{3}{*}{ Variable } & \multicolumn{6}{|c|}{ ED visit or Hospitalization within 1 year } \\
\hline & \multirow{2}{*}{$\begin{array}{l}\text { No ED visit/ } \\
\text { Hospitalization } \\
\quad(\mathbf{N}=\mathbf{5 0 6 4 )}\end{array}$} & \multirow{2}{*}{$\begin{array}{c}\text { ED visit/ } \\
\text { Hospitalization } \\
\text { within } 1 \text { year } \\
(\mathrm{N}=2674)\end{array}$} & \multicolumn{3}{|c|}{ OR \& $95 \%$ CI } & \multirow[b]{2}{*}{$P$ value } \\
\hline & & & OR & Lower & Upper & \\
\hline Age, y, mean $( \pm$ SD $)$ & $75.15( \pm 7.39)$ & $78.05( \pm 7.87)$ & 1.05 & 1.04 & 1.06 & $<.0001$ \\
\hline Male, n (\%) & $2175(43)$ & $1168(44)$ & 1.03 & 0.94 & 1.13 & 0.5375 \\
\hline \multicolumn{7}{|l|}{ MAGE survey, n (\%) } \\
\hline History of weight loss in last 3 months & $335(7)$ & $263(10)$ & 1.54 & 1.30 & 1.82 & $<.0001$ \\
\hline History of 2 or more falls in past year & $481(10)$ & $481(18)$ & 2.09 & 1.83 & 2.40 & $<.0001$ \\
\hline History of being hurt in a fall in past year & $528(11)$ & $402(15)$ & 1.52 & 1.32 & 1.74 & $<.0001$ \\
\hline History of difficulty with vision & $913(18)$ & $613(23)$ & 1.35 & 1.21 & 1.52 & $<.0001$ \\
\hline History of difficulty with hearing & $1935(39)$ & $1170(44)$ & 1.25 & 1.14 & 1.38 & $<.0001$ \\
\hline History of being sad or depressed & $469(9)$ & $395(15)$ & 1.72 & 1.49 & 1.98 & $<.0001$ \\
\hline History of unintended urinary leakage & $1994(40)$ & $1153(44)$ & 1.17 & 1.07 & 1.29 & 0.0011 \\
\hline $\begin{array}{l}\text { History of urinary leakage severe enough to } \\
\text { see treatment }\end{array}$ & $360(7)$ & $266(11)$ & 1.45 & 1.23 & 1.71 & $<.0001$ \\
\hline Rate your health compared to others: Worse & $192(4)$ & $240(9)$ & 2.55 & 2.10 & 3.10 & $<.0001$ \\
\hline
\end{tabular}

and combined outcomes based on the scoring system are shown in Table 4.

\section{DISCUSSION}

In this retrospective study of 7738 elderly patients presenting for outpatient primary care visits, we found that the MAGE questionnaire could be used to screen for common geriatric issues. We also found that in addition to advanced age, a self-report of worse health, history of falls, weight loss, and depressed mood in the MAGE questionnaire were significantly associated with hospitalization or ED visits within 1 year.

Previous studies have shown that geriatric screening and risk assessment can have high detection rates and help to identify high-risk elderly who may benefit from targeted interventions in different settings [9-11]. The results of this study are similar to other validated instruments that evaluate risk, but some important differences should be highlighted. The Ambulatory Care Group (ACG) system predicts utilization of ambulatory health services by classifying patients into one of $51 \mathrm{ACG}$ categories based on age, gender, and ICD9 coding diagnoses [4]. The Probability for Repeated Admissions (Pra), a well-validated screening tool derived from noninstitutionalized elderly patients, uses self-rated health, medical and social history, functional status, and previous pattern of health care utilization to predict repeated hospitalization over 4 years [2]. Other studies have used mailed questionnaires to determine predictors in this population quoting variable response rates $[3,12$, 13]. In our institution, screening instruments derived from administrative data on comorbid status and previous health care utilization, such as the Elder Risk Assessment have been successfully used to identify high-risk elderly who have previously been hospitalized [6, 7]. Although screening at the time of hospitalization or care transitions is valuable to prevent future events, there may be a risk of overestimating some risk factors while a patient is hospitalized. In contrast to these, the MAGE was completed as a part of the clinical care process at the time of their outpatient appointment by all communitydwelling elderly who sought primary care irrespective of their previous hospitalization status. It included patient self-report of geriatric symptoms as well as selfassessment of their health, and we found that their responses could be used to identify those at increased risk for $\mathrm{ED}$ visits and hospitalizations in 1 year. 
Table 3. Multivariable model for predictors of ED visit or hospitalization within 1 year of completing the MAGE

\begin{tabular}{|c|c|c|c|c|c|c|c|c|}
\hline \multirow[b]{3}{*}{ Standard Parameter } & \multicolumn{5}{|c|}{ From single run } & \multicolumn{3}{|c|}{ From simulations } \\
\hline & \multirow[b]{2}{*}{ Estimate } & \multirow[b]{2}{*}{ OR } & \multicolumn{2}{|c|}{$95 \% \mathrm{CI}$} & \multirow[b]{2}{*}{$P$ value } & \multirow[b]{2}{*}{$\begin{array}{c}\text { Estimate } \\
\text { (Average) }\end{array}$} & \multirow[b]{2}{*}{ OR } & \multirow[b]{2}{*}{ Score } \\
\hline & & & Lower & Upper & & & & \\
\hline Intercept & -1.367 & & & & $<.0001$ & & & \\
\hline Male & 0.080 & 1.08 & 0.97 & 1.21 & 0.1483 & 0.085 & 1.09 & \\
\hline Age $70-74$ & 0.287 & 1.33 & 1.14 & 1.56 & 0.0004 & 0.284 & 1.33 & 1.000 \\
\hline Age $75-79$ & 0.608 & 1.84 & 1.56 & 2.16 & $<.0001$ & 0.601 & 1.83 & 2.000 \\
\hline Age 80 or more & 0.826 & 2.29 & 1.98 & 2.64 & $<.0001$ & 0.822 & 2.28 & 2.000 \\
\hline $\begin{array}{l}\text { History of weight loss in last } 3 \\
\text { months }\end{array}$ & 0.210 & 1.23 & 1.02 & 1.49 & 0.0286 & 0.217 & 1.24 & 1.000 \\
\hline History of 2 or more falls & 0.487 & 1.63 & 1.38 & 1.93 & $<.0001$ & 0.486 & 1.63 & 2.000 \\
\hline History of being hurt in a fall & 0.105 & 1.11 & 0.94 & 1.32 & 0.2244 & 0.106 & 1.11 & \\
\hline History of difficulty with vision & 0.075 & 1.08 & 0.94 & 1.23 & 0.2681 & 0.073 & 1.08 & \\
\hline History of difficulty with hearing & 0.070 & 1.07 & 0.96 & 1.20 & 0.2102 & 0.068 & 1.07 & \\
\hline History of being sad or depressed & 0.272 & 1.31 & 1.11 & 1.55 & 0.0011 & 0.273 & 1.31 & 1.000 \\
\hline $\begin{array}{l}\text { History of unintended urinary } \\
\text { leakage }\end{array}$ & -0.053 & 0.95 & 0.85 & 1.06 & 0.3689 & -0.052 & 0.95 & \\
\hline $\begin{array}{l}\text { History of urinary leakage severe } \\
\text { enough to seek treatment }\end{array}$ & 0.149 & 1.16 & 0.96 & 1.41 & 0.1348 & 0.146 & 1.16 & \\
\hline Worse self-rated health & 0.748 & 2.11 & 1.70 & 2.63 & $<.0001$ & 0.760 & 2.14 & 2.000 \\
\hline
\end{tabular}

ED: Emergency department; MAGE: Mayo Ambulatory Geriatric Evaluation; OR: Odds Ratio; CI: Confidence Interval

On evaluating the predictors individually, we found that a self-report of worse health compared to peers was a strong predictor of hospitalization and/or ED visit in our population. Self-rated health is a well-known predictor for adverse outcomes in the elderly. Patient perception of their health status is an important component of the Pra instrument, a widely used geriatric tool that has been validated in several populations $[2,3,12,14,15]$. Selfrated health is also a component of the Vulnerable Elders Survey, a tool which was originally designed to identify elderly at higher risk of death and functional decline but has also been shown to predict increased health care utilization [16, 17]. A poor self-rated health may reflect medical complexity and greater need for health care services. Finding a worse self-rated health as a strong predictor of hospitalization and ED visits in the MAGE survey confirms its significance in the ambulatory population.
A history of 2 or more falls in the previous year was also an important risk for hospitalization and $\mathrm{ED}$ visits within 1 year. Falls are common. It is estimated that one in three adults aged 65 and older fall each year. Falls are a leading cause of injury and death from unintentional injury in patients over the age of 65 [18]. In 2010, 2.3 million nonfatal injuries among older adults were treated in the ED; of these 662,000 were hospitalized [19]. Recent research suggests that there may be primary care interventions that can reduce falling among the elderly [20]. Despite this, patients often do not discuss falls with their providers, and there is a lack of awareness among providers regarding inquiring about this important geriatric issue. The results of this study highlight the importance of falls in affecting health outcomes in the elderly. 
Table 4. Results of the score system from the logistic model to predict ED visits and/or hospitalizations within 1 year of completing MAGE

\begin{tabular}{lccccc}
\hline & \multicolumn{5}{c}{ MAGE Score } \\
\cline { 2 - 5 } & $\mathbf{0}$ & $\mathbf{1}$ & $\mathbf{2}$ & $\mathbf{3 +}$ & \\
Variable & $\mathbf{( N = 1 3 9 1 )}$ & $\mathbf{( N = 1 4 0 3 )}$ & $\mathbf{( N = 2 8 8 1 )}$ & $\mathbf{( N = 1 5 6 5 )}$ & $\boldsymbol{P}$ value \\
\hline ED visits, n (\%) & $216(16)$ & $283(20)$ & $895(31)$ & $695(44)$ & $<.001$ \\
Hospitalization, n (\%) & $170(12)$ & $214(15)$ & $684(24)$ & $562(36)$ & $<.001$ \\
ED visit/hospitalization, n (\%) & $289(21)$ & $358(26)$ & $1043(36)$ & $784(50)$ & $<.001$ \\
\hline
\end{tabular}

ED: emergency department; MAGE: Mayo Ambulatory Geriatric Evaluation

Another predictor for adverse outcomes in this study was a history of unintentional weight loss. Elderly are at risk for malnutrition [21]. Weight loss is an important component of many nutritional screening instruments such as the Subjective Global Assessment and the Mini Nutritional Assessment [22, 23]. Serious medical illness or psychiatric conditions may put patients at risk for malnutrition as well as adverse health outcomes. Weight loss is a component of the phenotype of frailty [24] which may also explain hospitalizations and ED visits. Unless screened for, neither the patient nor provider may bring up weight loss if the visit is for unrelated issues.

A history of feeling sad or depressed also remained significant. This is in concurrence with previous work that has shown a history of depressive symptoms to be associated with increased health care utilization $[25,26]$ and unplanned readmissions in the elderly [27]. Depressed mood may impact the ability to care for oneself which may lead to ED or hospitalization for these issues. A recent Morbidity and Mortality Weekly Report released by the Centers for Disease Control and Prevention reports a prevalence rate of $6.9 \%$ for any depression in US adults aged 65 and older [28]. We found that $11 \%$ of those completing the survey reported a sad or depressed mood, thus underscoring the need to screen for mood concerns in this population.

Lastly, in the current push to contain costs, research on elderly patients is often focused on survival and health care utilization. However, symptoms that affect quality of life also need to be addressed. A recent study has reported that although screening detected unidentified problems in general practice there were differences in the importance of problems as perceived by the provider and the patient [29]. The MAGE survey had high detection rates of common geriatric problems such as vision and hearing difficulties and urinary incontinence. While not significant predictors of an ED visit or hospitalization in this study, these issues are important for the quality of life for elderly patients, and unless screened for, they may be accepted by the patient as a part of aging and overlooked by providers.

\section{Strengths and limitations}

The MAGE survey was completed by patients at the time of their office visit as a part of their clinical care process. Thus, the information was accurate and up to date. Since completion of the survey was required as a part of the standardized rooming process without exclusions and a part of routine clinical care, we believe that the number of persons who did not complete the survey would be small. However, exact numbers for missing surveys are not available. Additional strengths include the number of patients and incorporation of patient self-report of their symptoms as predictors rather than billing or other administrative data. We performed quality checks at critical junctures to ensure accuracy and enhance validity.

A limitation of this study is its retrospective design; however, use of the unified EMR enhances the accuracy of the hospitalizations and ED visits. It is possible that some hospitalizations or ED visits were missed if patients sought care outside of our medical institution. Another weakness in the study is that despite changing demographics in the United States, the population in this county is largely Caucasian, and minority segments may not have been as well represented [30]. Our institution is a tertiary care center, but the Division of PCIM serves only the local population of Olmsted County, MN, and surrounding areas, thus minimizing referral bias. 
Medical comorbidity, functional status, and previous ED visits and hospitalization were not included as predictors in this study. Each of these is known to have significant impact on the outcomes we were studying, so this is a limitation of this study. The MAGE survey was a clinical questionnaire introduced to improve geriatric screening in our practice. To keep this process efficient and less burdensome to patients and providers, this survey did not require the patient to list their medical diagnoses or previous ED visits or hospitalizations. This study is a retrospective analysis of this process. The Charlson comorbid index scores and previous hospitalization and ED visits could be accessed from administrative databases and chart review. However, we opted to not use these since this information cannot be easily provided by the patient at the time of an office visit and our goal was to study the utility of MAGE as a patient-reported survey.

This is a single institution study. Further research is needed to validate these results and determine if they are applicable to other populations and different settings such as nursing homes and assisted living facilities. Future studies could be undertaken to explore whether such geriatric screening can be used to predict other adverse outcomes such as placement in a skilled nursing facility or fall-related injuries. Prospective research is also needed to explore if interventions on predictors such as falls and depression can impact outcomes.

In summary, with this retrospective study of 7738 elderly patients presenting for outpatient primary care visits, we found that responses to a short survey completed by the patient at the time of their office visit to screen for common geriatric conditions could also be used to assess risk for adverse outcomes such as ED visits or hospitalization within 1 year. A worse self-reported health, advanced age, and history of 2 or more falls strongly predicted ED visits and hospitalization within 1 year. Future studies are needed to confirm these results and explore their applicability in other settings.

\section{Acknowledgement}

We acknowledge receipt of the Mayo Clinic Department of Medicine Write-up and Publish (WRAP) grant for this manuscript.

\section{References}

[1] Centers for Medicare \& Medicaid Services (CMS). Affordable Care Act Update: Implementing Medicare Cost Savings (2010) [accessed May 22, 2013]. Available from: http:/www.cms.gov/apps/docs/acaupdate-implementing-medicare-costs-savings.pdf.
[2] Boult C, Dowd B, McCaffrey D, Boult L, Hernandez R, Krulewitch H (1993). Screening elders for risk of hospital admission. J Am Geriatr Soc, 41:811-817.

[3] Pacala JT, Boult C, Boult L (1995). Predictive validity of a questionnaire that identifies older persons at risk for hospital admission. J Am Geriatr Soc, 43:374-377.

[4] Weiner JP, Starfield BH, Steinwachs DM, Mumford LM (1991). Development and application of a populationoriented measure of ambulatory care case-mix. Med Care, 29:452-472.

[5] Lemke KW, Weiner JP, Clark JM (2012). Development and validation of a model for predicting inpatient hospitalization. Med Care, 50:131-139.

[6] Takahashi PY, Tung EE, Crane SJ, Chaudhry R, Cha S, Hanson GJ (2012). Use of the elderly risk assessment (ERA) index to predict 2-year mortality and nursing home placement among community dwelling older adults. Arch Gerontol Geriatr, 54:34-38.

[7] Crane SJ, Tung EE, Hanson GJ, Cha S, Chaudhry R, Takahashi PY (2012). Use of an electronic administrative database to identify older community dwelling adults at high-risk for hospitalization or emergency department visits: the elders risk assessment index. BMC Health Serv Res, 10:338.

[8] Rockwood K, Mitnitski A (2011). Frailty defined by deficit accumulation and geriatric medicine defined by frailty. Clin Geriatr Med, 27:17-26.

[9] Pepersack T, College of Geriatrics and the Belgian Society for Gerontology and Geriatrics (2008). Minimum geriatric screening tools to detect common geriatric problems. J Nutr Health Aging, 12:348-352.

[10] Boult C, Boult LB, Morishita L, Dowd B, Kane RL, Urdangarin CF (2001). A randomized clinical trial of outpatient geriatric evaluation and management. J Am Geriatr Soc, 49:351-359.

[11] Monteserin R, Brotons C, Moral I, Altimir S, San Jose A, Santaeugenia S, et al (2010). Effectiveness of a geriatric intervention in primary care: a randomized clinical trial. Fam Pract, 27:239-245.

[12] Pacala JT, Boult C, Reed RL, Aliberti E (1997). Predictive validity of the Pra instrument among older recipients of managed care. J Am Geriatr Soc, 45:614617.

[13] Dorr DA, Jones SS, Burns L, Donnelly SM, Brunker CP, Wilcox A, et al (2006). Use of health-related, quality-oflife metrics to predict mortality and hospitalizations in community-dwelling seniors. J Am Geriatr Soc, 54:667673.

[14] Boult C, Pacala JT, Boult LB (1995). Targeting elders for geriatric evaluation and management: reliability, validity, and practicality of a questionnaire. Aging (Milano), 7:159-164.

[15] Wagner JT, Bachmann LM, Boult C, Harari D, von Renteln-Kruse W, Egger M, et al (2006). Predicting the risk of hospital admission in older persons--validation of a brief self-administered questionnaire in three European countries. J Am Geriatr Soc, 54:1271-1276.

[16] Saliba D, Elliott M, Rubenstein LZ, Solomon DH, Young RT, Kamberg CJ, et al (2001). The Vulnerable 
Elders Survey: a tool for identifying vulnerable older people in the community. J Am Geriatr Soc, 49:16911699.

[17] McGee HM, O'Hanlon A, Barker M, Hickey A, Montgomery A, Conroy R, et al (2008). Vulnerable older people in the community: relationship between the Vulnerable Elders Survey and health service use. J Am Geriatr Soc, 56:8-15.

[18] Centers for Disease Control and Prevention, National Center for Injury Prevention and Control. Web - based Injury Statistics Query and Reporting System (WISQARS) [online]. Leading Causes of Death Reports, National and Regional, 1999 - 2010 [accessed May 22, 2013]. Available from: webappa.cdc.gov/sasweb/ncipc/leadcaus10_us.html.

[19] Centers for Disease Control and Prevention (CDC). Falls Among Older Adults: An Overview. [accessed May 22, 2013]; Available from: www.cdc.gov/homeandrecreationalsafety/falls/adultfall s.html.

[20] Michael YL, Whitlock EP, Lin JS, Fu R, O'Connor EA, Gold R (2010). Primary Care - Relevant Interventions to Prevent Falling in Older Adults: A Systematic Evidence Review for the U.S. Preventive Services Task Force. Ann Intern Med, 153:815-825.

[21] Persson MD, Brismar KE, Katzarski KS, Nordenstrom J, Cederholm TE (2002). Nutritional status using mini nutritional assessment and subjective global assessment predict mortality in geriatric patients. J Am Geriatr Soc, 50:1996-2002.

[22] Detsky AS, McLaughlin JR, Baker JP, Johnston N, Whittaker S, Mendelson RA, et al (1987). What is subjective global assessment of nutritional status? JPEN J Parenter Enteral Nutr, 11:8-13.

[23] Guigoz Y, Vellas B, Garry PJ (1996). Assessing the nutritional status of the elderly: The Mini Nutritional Assessment as part of the geriatric evaluation. Nutr Rev, 54:S59-65.

[24] Fried LP, Tangen CM, Walston J, Newman AB, Hirsch C, Gottdiener J, et al (2001). Frailty in older adults: evidence for a phenotype. J Gerontol A Biol Sci Med Sci, 56:M146-156.

[25] Rowan PJ, Davidson K, Campbell JA, Dobrez DG, MacLean DR (2002). Depressive symptoms predict medical care utilization in a population-based sample. Psychol Med, 32:903-908.

[26] Press Y, Tandeter H, Romem P, Hazzan R, Farkash M (2012). Depressive symptomatology as a risk factor for increased health service utilization among elderly patients in primary care. Arch Gerontol Geriatr, 54:127130.

[27] Marcantonio ER, McKean S, Goldfinger M, Kleefield S, Yurkofsky M, Brennan TA (1999). Factors associated with unplanned hospital readmission among patients 65 years of age and older in a Medicare managed care plan. Am J Med, 107:13-17.

[28] Centers for Disease Control and Prevention (CDC) (2010). Current Depression Among Adults --- United
States, 2006 and 2008. MMWR Morb Mortal Wkly Rep, 59:1229-1235.

[29] Piccoliori G, Gerolimon E, Abholz HH (2008). Geriatric assessment in general practice using a screening instrument: is it worth the effort? Results of a South Tyrol Study. Age Ageing, 37:647-652.

[30] U.S. Census Bureau, 2010 Census. Demographics and Census Data (2010) [accessed September 17, 2013];

Available from: http://www.co.olmsted.mn.us/ yourgovernment/demographics/Pages/default.aspx. 